\title{
SINGLE SAMPLE RECOGNITION BASED ON LPP
}

\author{
Qingqing $\mathrm{Li}^{1}$, Xueqiang $\mathrm{LV}^{1}$, Kai Zhang ${ }^{2}$ \\ ${ }^{1}$ School of Beijing Information Science \& Technology University, Beijing 100101, China \\ ${ }^{2}$ Beijing Chaoyang District Municipal Commission of City Administration and Environment, Beijing, \\ China
}

Keywords: locality preserving projection, S (2D) 2LPP, sub-feature, single sample.

\begin{abstract}
For a single sample face recognition problems, this paper propose a supervised two-way two-dimensional locality preserving projection method to extract the facial features, based on locality preserving projection. The recognition phase, this paper presents a method of subcharacteristics voting, In this method, firstly, make face image into blocks, each subset of blocks as a sub-feature and statistics weights of each sub-feature, finally, identify by voting. Experimental results on YaleB and Yale show that the presented method can achieve a certain degree of recognition accuracy.
\end{abstract}

\section{Introduction}

Face recognition technology is one of the biometric identification technologies, the main purpose is identification by visual characteristics of the human face. Face recognition involves several research areas, such as image processing, machine vision, pattern recognition and so on. With the development of computer technology and the wide range of application requirements, face recognition technology has become a hot topic, and has made great progress. The main current methods of face recognition are geometric features, feature subspace, neural network method, elastic matching method, hidden Markov model method and so on.

Subspace methods are PCA, LDA, ICA, etc.These are the traditional linear dimensionality reduction methods, and is good at global linear structure. Literature do principal component analysis for the face image training set, get feature subspace from transform matrix which is applied with singular value, project the training image and test image into subspace, then select classifier for face recognition. Literature extract feature subspace of image training sets using PCA, the training images and test images are projected in the subspace, the feature vectors are extracted and the statistical properties are calculated, adopt Bayesian decision based on minimum error rate to realize face recognition. Literatur propose an improved method which do 2DPCA reconstruction in class, deal the 2-dimensional image matrix directly, avoiding the high computational complexity which is caused by stretching the 1-dimensional vector. But the face features are usually characterized by a high nonlinear dimensionality reduction, using these methods will lost the intrinsic relationship hidden in the data. The study showed that the high nonlinear structures have low-dimensional manifold, manifold learning theory can solve nonlinear problems, and has been successfully applied. Rowels and Saul proposed locally linear embedding (LLE algorithm, which can be mapped high-dimensional data point into a global low-dimensional coordinate system, is still maintained after the near-relational mapping high-dimensional, so that we can effectively retain the nonlinear structure of data, but also translational and rotational invariance. Literature combine the linear subspace method and manifold learning method, assign a new face recognition algorithm: Local Maximum Margin Linear Distinct Embedding, but the identification result is not good when apply single subspace analysis into large-scale face database.

Tenenbaum et proposed isometric mapping (ISOMAP) algorithm, which will build a nearest neighbor graph by data points, the shortest route instead of Euclidean distance, and then after multidimensional scaling analysis (MDS) process, get low-dimensional coordinates which can be embeddable in high-dimensional space. On this basis, there are some improved algorithms, such as Laplace feature mapping algorithm (LE), the Hessian LLE algorithm (HLLE), the local tangent 
space sorting algorithm (LTSA), etc. Traditional manifold learning algorithm does not get projection matrix from high-dimensional to low-dimensional, so extracting features directly from new samples is difficulty. He et put forward a locality preserving projections algorithm (LPP) to solves this problem.

This article is based on LPP features, and improved projection matrix, using bi-dimensional supervised LPP method to extract features of face images and using child features of the proposed voting method to classify, after experimental verification, the method in the paper is more effective than LPP method.

\section{LPP}

The basic idea of LPP is to obtain an transformation matrix, can project high-dimensional data set onto a low-dimensional data set, and ensure that adjacent data in original data set are also adjacent to each other in projection data set. Algorithm as follows:

Construction of the adjacent map. The map can be an weighted undirected graph with nodes. There are two ways to determine whether there is a connection between the points $i$ and $j$ : one is $\varepsilon$ near, that is, the distance between $x_{i}, x_{j}$ meet $\left\|x_{i}-x_{j}\right\|^{2}<\varepsilon$; another method is k near, that is, node $i$ is one of the $k$ adjacent nearest nodes of node $j$, or node $j$ is one of the $k$ nearest adjacent nodes of node $i$.

Dtermine the edge value matrix $S$ of the edge in adjacent diagram $G$. When there is no edge between the points $i$ and $j$, the weight value is 0 ,otherwise, the weight calculation methods are: one is simple, that is $S_{i j}=1$, another one is the thermonuclear function, that is

$S_{i j}=\exp \left(\frac{-\left\|x_{i}-x_{j}\right\|^{2}}{2}\right)$

Feature projection. Calculate the feature and feature vector.

$X^{T} L X \omega=\lambda X^{T} D X \omega$

Among them $D$ is a diagonal matrix whose elements is combined by the sum of row or column items, that is $D_{i i}=\sum_{j} S_{i j} . L$ is the Laplacian matrix, $L=D-S$.

Projection matrix $W=\left\{\omega_{1}, \omega_{2} \cdots \omega_{d}\right\}, W_{i}$ is the corresponding feature vector of feature value $\lambda_{i}$, and $\lambda_{1} \leq \lambda_{2} \leq \cdots \leq \lambda_{d}$.

$y_{i}=x_{i} W$

The projection matrix $W$ in LPP algorithm need meet the contraints

$\left\{\begin{array}{c}\min \sum_{i, j} S_{i j}\left\|y_{i}-y_{j}\right\|^{2} \\ y^{T} D y=1 \Rightarrow \omega^{T} X^{T} D X \omega=1\end{array}\right.$

And

$\sum_{i, j} S_{i j}\left\|y_{i}-y_{j}\right\|^{2}=2 \omega^{T} X^{T} L X \omega$

The corresponding Lagrange function of min question is 
$g(\omega, \lambda)=\omega^{T} X^{T} L X \omega+\lambda\left(1-\omega^{T} X^{T} D X \omega\right)$

Set the first detivative value of $W$ is 0 , then formular (2) can be got.

\section{Supervised Bi-dimensional Locality Preserving projections}

Inspired by two-way principal component analysis, we propose a supervised two-way twodimensional locality preserving projection. The projection formula of S(2D)2LPP as follow:

$y_{i}=V^{T} X_{i} U$

Among them, $V$ is the projection matrix in the vertical direction, and $U$ is the projection matrix in the horizontal direction. The general 2DLPP is the horizontal projection, the constraints on vertical direction are:

$$
\begin{gathered}
\left\{\begin{array}{c}
\min \sum_{i, j} S_{i j}\left\|y_{i}-y_{j}\right\|^{2} \\
y D y^{T}=1 \Rightarrow \omega X\left(D \otimes I_{n}\right) X^{T} \omega^{T}=1
\end{array}\right. \\
\sum_{i, j} S_{i j}\left\|Y_{i}-Y_{j}\right\|^{2}=2 v X\left(L \otimes I_{n}\right) X^{T} v^{T}
\end{gathered}
$$

Among them

$$
S_{i j}=\left\{\begin{array}{cc}
\theta \exp \left(\frac{-\left\|X_{i}-X_{j}\right\|^{2}}{2}\right) & i, j \text { adjacent and similar } \\
(1-\theta) \exp \left(\frac{-\left\|X_{i}-X_{j}\right\|^{2}}{2}\right) & i, j \text { adjacent and unsimilar } \\
0 & i, j \text { unadjacent }
\end{array}\right.
$$

The corresponding Lagrange function of min question is

$$
g(v, \lambda)=v X\left(L \otimes I_{n}\right) X^{T} v^{T}+\lambda\left(1-v X\left(D \otimes I_{n}\right) X^{T} v^{T}\right)
$$

Set the first detivative value of $W$ is 0 , then

$$
X\left(L \otimes I_{n}\right) X^{T} v^{T}=\lambda X\left(D \otimes I_{n}\right) X^{T} v^{T}
$$

(12)Face recognition algorithm based on S (2D)

2LPP is divided into three phases:

(1) Calculate the projection matrix. Based on the theory of general learning framework, using S(2D)2LPP algorithm to get projection matrix from the training image set.

(2) Feature extraction. Extracting features from the image set according to the formula (12).

(3) Identification. Extracting features from unknown image, and calculating the similarity distance between the unknown image and the images in the dataset, using the nearest neighbor classifier for classification.

\section{Images Classification Method}

In this paper, a face image is divided into $m$ sub-graphs, which constitute a sub-graph set, each non-empty set is the set of sub-features, so a total of face image have $n$ features, including $n=2^{m}-1$. 


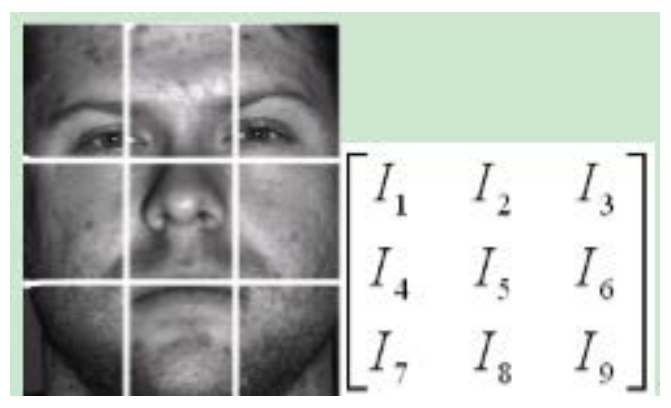

Figure 1. Block method

Calculating sub-features weights by statistical method, for a train image set which has $S$ human and each one has $t$ images, follow these steps:

Step1: dividing the image into $m$ sub-graph, constitute sub-features $X_{i}, i=1 \cdots n$.

Step2: constituting a sub-graph set by sub-graph corresponding to each training image, calculating each projection matrix of sub-graph and calculating each image's features.

Step3: combining each sub-graph's features into a bigger vector, and setting it as the image features.

Step4: doing multi-group random testing. Statistic the weight of each sub-feature to classification as its weight.

Random testing procedure is as follows:

(1) The training test totally has $S$ people, selecting randomly one image as the known image for everyone, and then selecting randomly 30 percentage images from the excess images as the unknown images.

(2) Using each sub-feature as the whole image's feature respectively, calculating the distance between unknown image and each known images with k adjacent nearest way, when one of the $k$ images is from the same people with the unknown image, then the child feature counter is incremented.

$\operatorname{sum}_{i}=\left\{\begin{array}{cc}\operatorname{sum}_{i}+1 & i \text { is one of } k \text { adjacent nearrest } \\ \operatorname{sum}_{i} & \text { otherwise }\end{array}\right.$

(3) after multi-group random testing, calculating each sub-feature weight

$w_{i}=\frac{\text { sum }_{i}}{\text { Count }}$

Among them, Count is the total number of simulation testing.

Classification process based on image sub-feature as follow:

(1) blocking a known image, and then calculating each sub-image feature, combining each sub feature into a feature vector

(2) To an unknown image to be detected, the first step is blocking image, extracting sub-feature's feature vector.

(3) Calculating the unknown image and the known image's corresponding sub-feature distance, using $k$ neighboring ballot, vote the ${ }^{w_{i}}$ to its $k$ closest known images.

(4) statistica each known's votes, and set that the image with most votes is same with the unknown image.

\section{Experiment and Analysis}

In this experiment, the total number of images is $120 \times 120$, and each face image will be divided into $3 \times 3$ sub-graph, subgraph of size $60 \times 60$, step is 30, the number of sub-features is 511 . Setting the YaleB image library as training library to calculate each sub-graph projection matrix and wach 
sub-feature weight. In Yale face image library, selecting one image as known image for each people, the excess images are setting as unknown testing images.

The experiment of $\mathrm{k}$ choose image classification method based on image sub-feature uses $k$ nearest in random testing and classification process. In this experiment, the main aim is to observe the impact of recog

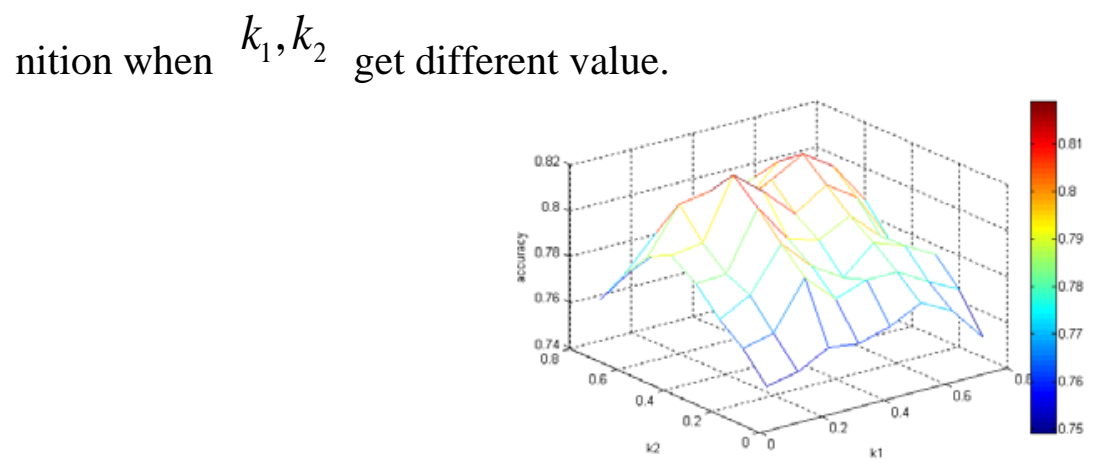

Figure 2. Experiment of $\mathrm{k}$ selection

What can see from Image 2 is that when $k_{1}=0.5, k_{2}=0.3$, the recognition result is the best.

Contrast experiments with different feature extraction methods. Using LPP, 2DLPP and S (2D)2LPP respectively to extract features.

From Figure 3 can see that the recognition method which is combined with sub-feature can get better recognition result. but when the scope of the interference region up to three, ie one third of the total, or more, the identify result is close to the original method or even lower.

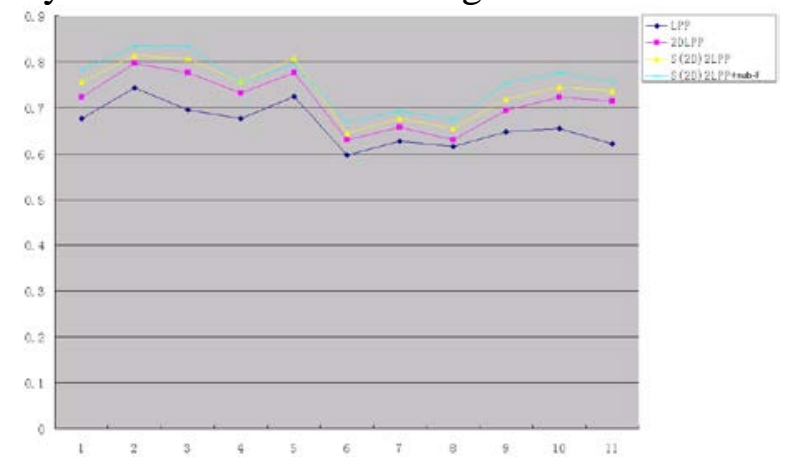

Figure 3. Yale face library contrast experiment

\section{Conclusion}

Based on locality preserving projection method, supervised bi-dimensional locality preserving projections method is proposed in this paper, and combining with the sub-feature voting method to classification. By experimental verification, the method is better than the traditional locality preserving projections in recognition. When there are disturbances in the face image and distributed in multiple sub-figure, the recognition result is not good, so how to eliminate interference and border issues in this regard is the next key research.

\section{Acknowledgements}

This work is supported by National Natural Science Foundation of China under Grants No. 61271304, Beijing Natural Science Foundation of Class B Key Project under Grants No. KZ201311232037, and Beijing University Innovation Team Building and Teacher Occupation Development Project under Grants No. IDHT20130519. 


\section{References}

[1] Belkin M, Niyogi P. Laplacian eigenmaps for dimensionality reduction and data representation. Neural Computation, Vol. 2003,15(6):1373-1396.

[2] Chen Lei. Face Recognition Algorithm Base on Linear Subspace and Manifold Learning (MS., Xian University of Electronic Science and Technology, China, 2014), p.10.

[3] Chen Sibao, Zhao Haifeng, Kong Min, etc. 2D-LPP: A two-dimensional extension of locality preserving projections [J]. Neurocomputing [C], 2007,70 (46): 912 921.

[4] Chen Yunachun. Face Recognition Based on Principal Component Analysis Matrix (MS., Wuhan University of Technology, China 2012), P.5.

[5] Daoqiang Zhang, Zhihua Zhou. Two-dircetional two-dimensional PCA for Efficientface representation and recognition [J]. Neurocomputing, 2005,69: 224 231.

[6] Donoho D L, Grimes C. 2003.Hessian eigenmaps: Locally linear embedding techniques for high-dimensional data[J].Proceedings of the National Academy of Sciences of the United States of America, 2003,100(10):5591-5596.

[7] He X F, Niyogi P. Locality Preserving Projections [A]. In: proceedings of Neural Information Proeessing System [C].Vancouver, Canada, 2003:153-160.

[8] Quan Xinghui, Mou Haiwei, Lv Xiuli, Zhang Hua. Face Recognition Based on PCA and Bayesian Decision [J]. Optical Instruments, 2014.36 (2): 122-125.

[9] Roweis S, Saul L K. Nonlinear dimensionality reduction by locally linear embedding [J].Seience, 2000, 290 (5500):2323-2326.

[10] Tenenbaum J B, de Silva V, Langford J C.A global geometric frame-work for nonlinear dimensionality reduction [J]. Seience, 2000, 290(5500):2319-2323.

[11] Xianghui Quan, Li Yu, Chunyue Ji. Face Recognition Based on Principal Component Analysis [J]. Science Technology and Engineering, 2010.10 (24): 6063-6065.

[12] Zhang Z Y, Zha H Y. Principal manifolds and nonlinear dimensionality reduction via tangent space alignment .SIAM Journal of Scientific Computing, 2004, 26 (1):313-338. 